\title{
The Sensory Insular Cortex Mediates the Stress-Buffering Effects of Safety Signals But Not Behavioral Control
}

\author{
John P. Christianson, Alexander M. Benison, Joshua Jennings, Emilee K. Sandsmark, Jose Amat, Richard D. Kaufman, \\ Michael V. Baratta, Evan D. Paul, Serge Campeau, Linda R. Watkins, Daniel S. Barth, and Steven F. Maier \\ Department of Psychology, Center for Neuroscience, University of Colorado, Boulder, Colorado 80309
}

\begin{abstract}
Safety signals are learned cues that predict stress-free periods whereas behavioral control is the ability to modify a stressor by behavioral actions. Both serve to attenuate the effects of stressors such as uncontrollable shocks. Internal and external cues produced by a controlling behavior are followed by a stressor-free interval, and so it is possible that safety learning is fundamental to the effect of control. If this is the case then behavioral control and safety should recruit the same neural machinery. Interestingly, safety signals that prevented a behavioral outcome of stressor exposure that is also blocked by control (reduced social exploration) failed to inhibit activity in the dorsal raphé nucleus or use the ventromedial prefrontal cortex, the mechanisms by which behavioral control operates. However, bilateral lesions to a region of posterior insular cortex, termed the "sensory insula," prevented the effect of safety but not of behavioral control, providing a double-dissociation. These results indicate that stressor-modulators can recruit distinct neural circuitry and imply a critical role of the sensory insula in safety learning.
\end{abstract}

Key words: rat; learned safety; conditioned inhibition; anxiety; somatosensory cortex; auditory cortex; evoked potentials

\section{Introduction}

Variables that modulate the impact of stressors on the development of psychological and physical disorders have received considerable research attention. Behavioral control (the real or perceived ability to alter the onset, termination or intensity of a stressor) blunts many of the behavioral and physiological consequences of the stressor (Mineka and Hendersen, 1985; Maier and Watkins, 1998; Minor and Hunter, 2002) and the neural substrates of behavioral control are well known (Maier and Watkins, 2005; Maier et al., 2006). The fear conditioned to cues present during stress is among the consequences modulated by control, with controllable producing less fear conditioning than equal amounts of uncontrollable stressors (Mineka et al., 1984). Interestingly, the presence of a "safety signal" during the stress experience also reduces the fear conditioned by that experience (Mineka et al., 1984) and mimics several effects of behavioral control beyond the reduction of conditioned fear (Weiss, 1971; Maier and Keith, 1987; Jackson and Minor, 1988; Minor et al., 1990). The term, safety signal, refers to the fact that stimuli that are unpaired with an aversive stimulus come to inhibit fear. Thus, for example, stimuli that regularly and immediately follow an aversive stimulus, and so are as far from the next aversive stimulus as is possible, become inhibitors of fear (Maier et al., 1976).

It has been argued that the effect of behavioral control can be

Received Sept. 5, 2008; revised 0ct. 21, 2008; accepted Nov. 7, 2008.

This work was supported by National Institutes of Health Grants MH050479 and NS36981, a seed grant from the University of Colorado Council on Research and Creative Work, and the University of Colorado Undergraduate Research Opportunity Program.

Correspondence should be addressed to John P. Christianson, Department of Psychology, Center for Neuroscience, UCB 345, Boulder, C0 80309. E-mail: john.christianson@colorado.edu.

DOI:10.1523/JNEUROSCI.4270-08.2008

Copyright $\odot 2008$ Society for Neuroscience 0270-6474/08/2813703-09\$15.00/0 reduced to a case of safety learning because interoceptive feedback cues generated by the stress controlling behavioral response come to signal safety (Weiss, 1971; Mineka et al., 1984; Maier and Keith, 1987; Jackson and Minor, 1988; Minor et al., 1990). If this is so then safety and behavioral control should recruit the same neural substrates. Behavioral control operates, at least in part, by inhibiting stressor-induced activation of serotonin (5-HT) neurons in the caudal dorsal raphé nucleus (DRN) (for review see Maier and Watkins, 2005) and the inhibition is provided by the ventromedial prefrontal cortex (vmPFC) efferents that terminate on GABA interneurons in the DRN (Maier et al., 2006). In the present experiments provision of a safety signal prevented later stress-induced anxiety-like behavior in a juvenile social exploration test, but safety signals did not inhibit the DRN, as does behavioral control. Furthermore, inhibition of the vmPFC by intracerebral microinjection of muscimol, a procedure known to eliminate the effects of behavioral control (Christianson et al., 2008b), had no effect on the safety signal. These data suggested that safety and control recruit distinct neural substrates.

Safety signal conditioning requires detection of coincident stimuli from multiple sensory modalities to determine under which circumstances to inhibit fear. The posterior insula, termed here the "sensory insula" ( $\mathrm{Si}$ ), has access to somatosensory information (Sudakov et al., 1971; Robinson and Burton, 1980a,b,c; Mufson and Mesulam, 1982; Shi and Cassell, 1998b; Flynn, 1999; Remple et al., 2003; Benison et al., 2007), a somatotopically organized body representation (Benison et al., 2007), exhibits convergent responses to simultaneous multisensory stimulation (Rodgers et al., 2008), and afferent intracortical and thalamocortical (Shi and Cassell, 1998b) and efferent amygdala connectivity (McDonald et al., 1999). These characteristics led us to hypothesize that $\mathrm{Si}$ would be involved in safety. Intriguingly, Si lesions 
made before stress prevented the effect of safety signals, but not of behavioral control.

\section{Materials and Methods \\ Rats}

Adult (60- to 70-d-old and weighing 275-350 g at the time of testing) and juvenile (28- to 32-d-old and weighing 90-100 g at the time of testing) male Sprague Dawley (Harlan) rats were used in all experiments. Rats were housed in plastic tub cages, 2 rats/cage with free access to food and water. The vivarium maintained a $12 \mathrm{~h}$ light/dark cycle with lights on at 7:00 A.M. All behavioral procedures were conducted in the first $5 \mathrm{~h}$ of the light cycle and the experimental protocols were reviewed and approved by the University of Colorado Institutional Animal Care and Use Committee.

\section{Tail shock and safety signal procedures}

Inescapable tail shocks (ISs) were administered in clear acrylic restraining tubes that were placed in a sound-attenuating chamber with a houselight (60 lux). Scrambled electric shock ( $1.6 \mathrm{~mA}$ ) was delivered to the tail through copper electrodes augmented with electrolyte paste by a Precision Regulated Animal Shocker operated by a LabLinc computer interface and Graphic State 3.0 software (Coulbourn Instruments). For groups Safe and Random, tail shocks were administered on a variableinterval $60 \mathrm{~s}$ schedule (VI-60; range $=20-140 \mathrm{~s}$ ). Shock duration varied between trials and averaged $5 \mathrm{~s}$ with a range of $1-10 \mathrm{~s}$. In the Safe group, a $5 \mathrm{~s}$ chamber blackout began with the termination of each shock. In the Random group the house light turned off for $5 \mathrm{~s}$ on a VI-60 s schedule that ran independent of the shock schedule. In the first experiment, two additional groups received tail shock. In one, rats were exposed the same schedule as the Random group but the house light remained on throughout the session (Variable). In the other, rats received 100, $5 \mathrm{~s}$ shocks with the house light on throughout the session (Fixed). This group was included because it is the stress induction procedure we have used recently (Christianson et al., 2008a,b). Rats in the home cage control group (HC) were left undisturbed in their home cages and in the first experiment, an additional home cage group (HC-Random) was left in the home cage but exposed to 100,5 s blackouts on a VI- $60 \mathrm{~s}$ schedule in a room adjacent to the stress room. The temporal arrangement of these shock and signal conditions is represented in Figure $1 \mathrm{~A}$. In the last experiment, 100 escapable tail shocks (ESs) were administered in acrylic wheel-turn boxes in which turning the wheel terminated the shock. The response requirement increased as rats became proficient and shock intensity was $1.0 \mathrm{~mA}$ for the first 33 trials, $1.3 \mathrm{~mA}$ for the next 33 trials, and 1.6 for the remaining 34 trials. These parameters have been used extensively (Amat et al., 2005) and serve to maintain escape behavior throughout the session. Each ES rat was yoked to an IS rat which received a tail shock of equal duration, but turning the wheel had no effect.

\section{Social exploration test}

Each rat was allocated a standard plastic tub cage with a wire lid and bedding located in a designated testing room. Twenty-four hours after stress, rats were placed in the test cage and $60 \mathrm{~min}$ later a naïve juvenile was added. An observer who was blind to the experimental treatment timed the exploratory behaviors initiated by the adult rat to the juvenile including sniffing, pinning and allogrooming. In experiments involving surgery all rats were given a prestress social exploration test to identify possible effects of the surgery on social exploration and to serve as a baseline when unstressed controls were not included (Experiment 3 ).

\section{Extracellular serotonin detection in the dorsal raphe nucleus}

Under ketamine $(71 \mathrm{mg} / \mathrm{kg}$ body weight), xylazine $(14 \mathrm{mg} / \mathrm{kg})$ and acepromazine $(2.4 \mathrm{mg} / \mathrm{kg})(\mathrm{K}-\mathrm{X}-\mathrm{A})$ anesthesia microdialysis CMA 12 guide cannulas (Carnegie Medicine) were aimed at the caudal DRN (AP $-8.3, \mathrm{LM} \pm 0, \mathrm{DV}-5.0$ ). A plastic screw cap from a $15 \mathrm{ml}$ conical centrifuge tube, with the center of the lid portion removed, was fixed to the skull surrounding the guide cannulas. At the time of dialysis, a portion of the centrifuge tube, open at both ends, was screwed into the cap to protect the cannula and tubing. $12-14 \mathrm{~h}$ before microdialysis began rats were transferred to a room maintained on the same light cycle as the vivarium and placed in plastic bowls with bedding, food and water. Mi-

\section{A Shock and Signal Conditions}

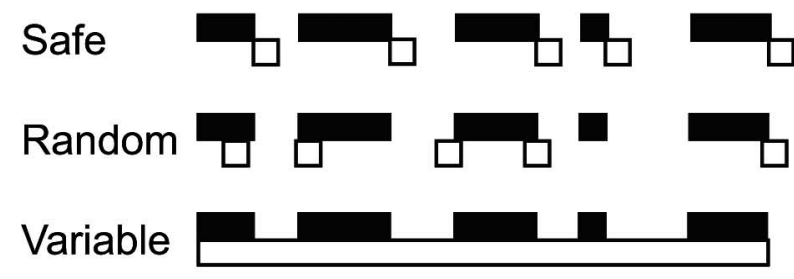

Fixed
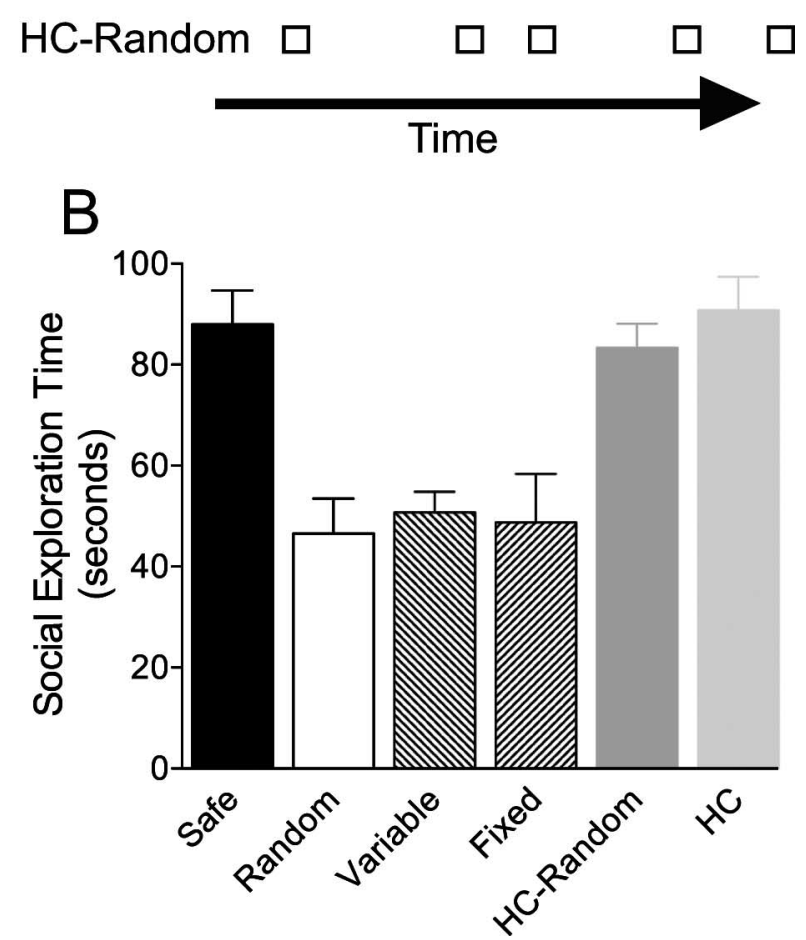

Figure 1. The effect of different shock and signal conditions on social exploration. $A$, Schematic illustration of the different shock and safety signal conditions used. Black filled bars represent the occurrence of tail shock, and unfilled boxes indicate the occurrence of a safety signal ( 5 s chamber blackout) over time. $\boldsymbol{B}$, Mean (+SEM) time spent exploring the juvenile conspecific in a 3 min test given $24 \mathrm{~h}$ after 100 tail shocks. Group designations indicate the conditions of previous tail shock (A) (see Materials and Methods, Tail shock and safety signal procedures).

crodialysis probes (CMA 12, MW cutoff $20 \mathrm{kDa}, 1 \mathrm{~mm}$ ) were inserted into the guide cannula and artificial CSF (ACSF: $145 \mathrm{~mm} \mathrm{NaCl}, 2.7 \mathrm{~mm}$ $\mathrm{KCl}, 1.2 \mathrm{~mm} \mathrm{CaCl}$ ) was perfused through the probes by a CMA infusion pump at a flow rate of $0.2 \mu \mathrm{l} / \mathrm{min}$. After $12 \mathrm{~h}$, the flow rate increased to 1.5 $\mu \mathrm{l} / \mathrm{min}$. After a $90 \mathrm{~min}$ equilibration period, 4 baseline samples were collected at $20 \mathrm{~min}$ intervals. Rats were transferred to acrylic restraint boxes with uninterrupted flow and the stress session began. Dialysates were collected manually at 20 min intervals throughout the stress session and for an additional $60 \mathrm{~min}$ after stress. Samples were placed in a $-80^{\circ} \mathrm{C}$ freezer until analysis with high-pressure liquid chromatography. 5-HT was measured using an ESA 5600A Coularray detector with an ESA 5014B analytic cell and an ESA 5020 guard cell. The column was an ESA $\mathrm{MD}-150$ maintained at $26^{\circ} \mathrm{C}$. Mobile phase was ESA buffer MD-TM. The analytical cell potentials were kept at -75 and $+250 \mathrm{mV}$ and the guard cell at $+300 \mathrm{mV}$. Dialysate $(23 \mu \mathrm{l})$ was injected using an ESA $542 \mathrm{au}-$ tosampler that kept the dialysates at $6^{\circ} \mathrm{C}$. External standards were run daily to quantify 5-HT. 


\section{Fos/5-HT immunohistochemistry}

Tissue preparation. Two hours after the end of the stress session rats were deeply anesthetized with sodium pentobarbital $(50 \mathrm{mg} / \mathrm{kg}$, i.p.) and transcardially perfused with $200 \mathrm{ml}$ of heparinized saline and then $200 \mathrm{ml}$ of $4 \%$ paraformaldehyde. Brains were collected and postfixed in paraformaldehyde for $24 \mathrm{~h}$ and then transferred to $30 \%$ sucrose cryoprotectant. $35 \mu \mathrm{m}$ sections were collected on a freezing cryostat $\left(-20^{\circ} \mathrm{C}\right)$ and stored in cryoprotectant at $4^{\circ} \mathrm{C}$.

Immunohistochemistry. Immunohistochemical staining for Fos was previously described (Grahn et al., 1999) using the avidin-biotinhorseradish $(\mathrm{ABC})$ method. In brief, Fos staining was conducted using a rabbit polyclonal primary antibody (1:15,000; Santa Cruz Biotechnology) and biotinylated goat anti-rabbit secondary antibody (Jackson ImmunoResearch)a nd visualized with 3,3'-diaminobenzine (DAB) and nickel chromogens. Serotonin (5-HT) staining used rabbit 5-HT primary antibody (1:10,000; ImmunoStar) and nonbiotinylated goat antirabbit IgG (Jackson ImmunoResearch) as the secondary antibody. 5-HT was visualized with peroxidase anti-peroxidase and DAB.

Fos/5-HT quantification. Under bright-field microscopy, an observer, blind to treatment, quantified the number of 5-HT-containing cells and the number of cells containing both 5-HT and Fos in two consecutive sections of the caudal DRN located $8.3 \mathrm{~mm}$ posterior to bregma. The average obtained from the two sections was used for statistical analysis. 5-HT-containing cells appeared as reddish-tan particles and 5-HT/Fos cells appeared as reddish-tan neurons containing black ovoid nuclei. The caudal DRN and examples of Fos, 5-HT and Fos/5-HT-immunoreactive cells are shown in Figure $2 B$ and $C$.

\section{Ventromedial prefrontal cortex cannulation}

Surgical preparation. Under isoflurane anesthesia (3\% in oxygen), dual guide cannula (26 g, $1 \mathrm{~mm}$ center-to-center distance; Plastics One) were implanted so that injector tips would reach the border between infralimbic and prelimbic cortices ( $\mathrm{AP}+2.9, \mathrm{LM} \pm 0.5, \mathrm{DV}-2.9$ from bregma and dura). Cannulas were fixed to the skull with screws and acrylic cement. A stylet was placed in the cannula extending $1 \mathrm{~mm}$ below the tip of the guide. Each rat received prophylactic antibiotic, $0.25 \mathrm{ml}$ of Twin-Pen (AgriLabs) per kg body weight (s.c).

Microinjections. Rats were gently restrained in a towel and a microinjector that extended $1 \mathrm{~mm}$ beyond the cannula tip ( $33 \mathrm{~g}$; Plastics One) was inserted. Muscimol, $500 \mathrm{ng} / \mathrm{side}$, in $0.5 \mu \mathrm{l}$ of $0.9 \%$ saline or saline alone was injected at a rate of $1 \mu \mathrm{l} / \mathrm{min}$; injectors remained in place for 2 min to permit diffusion. This method is identical to the one we have previously reported to prevent the stress-buffering effect of escapable stress on later social exploration (Christianson et al., 2008b). At the end of each experiment rats were overdosed with sodium pentobarbital and brains processed for cresyl violet verification of cannula placement using standard histological procedures.

\section{Excitotoxic sensory insular lesions}

Surgical preparation. Rats were anesthetized using subcutaneous injections of K-X-A and placed on a regulated heating pad to maintain normal body temperature $\left(37^{\circ} \mathrm{C}\right)$. Rats were placed in a stereotaxic frame and an incision was made over the temporalis muscle, which was bluntly dissected revealing the squamosal and frontal bones. Two burr holes, one, 4 $\mathrm{mm}$ rostral and $1 \mathrm{~mm}$ dorsal and another $3 \mathrm{~mm}$ rostral to the foramen located on the squamosal bone dorsal to the connection of the zygomatic arch allowed for injection of $0.28 \mu \mathrm{l}$ of NMDA (5\%) solution, in $.01 \mathrm{M}$ PBS at a depth of 600 and $300 \mu \mathrm{m}$ by a microinjector (Nanoliter 2000, World Precision Instruments) mounted on a stereotaxic positioner. After the injection the burr holes were filled with heated $\left(35^{\circ} \mathrm{C}\right)$ paraffin wax $(95 \%)$ and mineral oil (5\%) solution and cemented in place with dental cement. The animals were sutured and monitored closely for the next $7 \mathrm{~d}$. Postoperative and maintenance doses of Buprenex $(0.01 \mathrm{mg} / \mathrm{kg})$ and $9 \%$ saline $(10 \mathrm{cc})$ were administered after recovery from anesthesia. Rats were allowed 2 weeks of postoperative recovery before behavioral testing. Sham rats received identical procedures, although no NMDA was injected.

Electrophysiological verification. After behavioral testing all rats underwent electrophysiological verification of the lesion. Rats were anesthetized with K-X-A and placed on a regulated heating pad to maintain
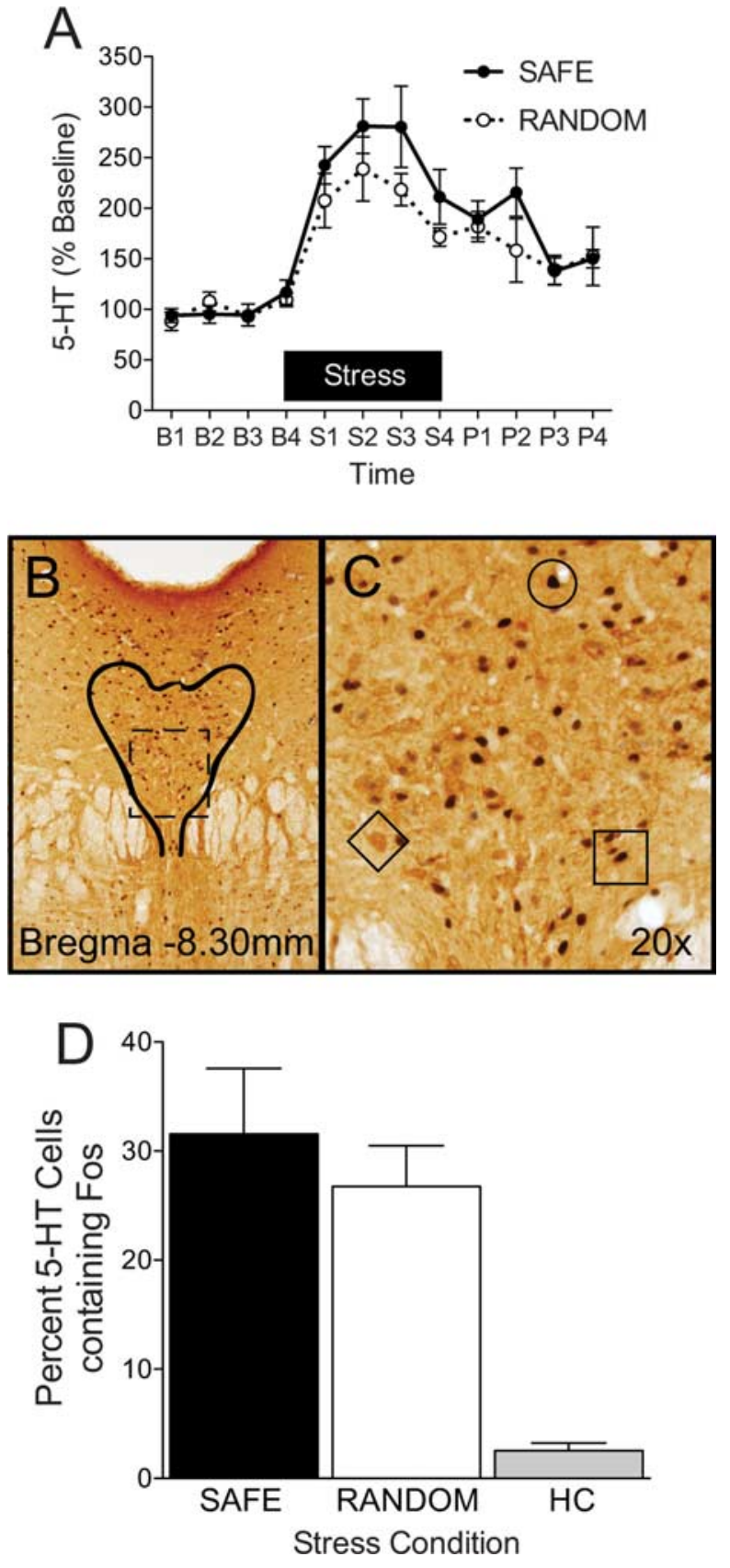

Figure 2. DRN 5-HT responses to inescapable tail-shock stress with either Safe or Random signals. A, Mean ( \pm SEM) levels of extracellular 5 -HT expressed as percentage of baseline. Samples were collected at $20 \mathrm{~min}$ intervals before shock (B1-4), during shock (S1-4) and after shock (P1-4). The black bar indicates the duration of the 100 tail shocks. $B$, $10 \times$ photomicrograph of the caudal DRN (Bregma $-8.30 \mathrm{~mm}$ ). Solid line illustrates the border of the DRN and the dashed box represents the field magnified on the right. C, $20 \times$ photomicrograph within the DRN illustrating neuronal soma stained positive for 5 -HT (enclosed in a diamond), Fos (enclosed in a circle) and double-labeled Fos and 5-HT (enclosed in a square). D, Mean (+SEM) percentage of neurons double-labeled for Fos and 5-HT in the caudal DRN. Filled circles/bar indicate stress with safety signals and open circles/bar indicate stress with random signals.

normal body temperature $\left(37^{\circ} \mathrm{C}\right)$. Anesthesia as maintained throughout the procedure so that the corneal and flexor withdrawal reflexes were weak. A bilateral craniotomy was performed over both hemispheres extending from bregma to $3 \mathrm{~mm}$ rostral of $\lambda$ and from the midsagittal suture past the lateral aspect of the temporal bone, exposing a maximal area of the surgically accessible hemisphere. The dura was reflected and the exposed cortex regularly doused with Ringer's solution containing $\mathrm{NaCl} 135 \mathrm{~mm}$; KCl $3 \mathrm{~mm}$; $\mathrm{MgCl} 2 \mathrm{~mm}$; and $\mathrm{CaCl} 2 \mathrm{~mm}, \mathrm{pH} 7.4$ at $37^{\circ} \mathrm{C}$. 
Epipial maps of somatosensory, auditory, and visual evoked potentials (SEP, AEP and VEP, respectively) were recorded using a flat multielectrode array consisting of 256 stainless steel wires in a $16 \times 16$ grid (tip diameter: $\sim 100 \mu \mathrm{m}$; interelectrode spacing: $400 \mu \mathrm{m}$ ) covering a $6 \times 6$ $\mathrm{mm}$ area of the right hemisphere in a single placement. The array was pressed against the cortex with sufficient force to establish contact of all electrodes. The required pressure had no effect on evoked potential amplitude, poststimulus latency, or morphology when compared with potentials recorded previously with more lightly placed small arrays (Benison et al., 2007). Recordings were referenced to a stainless steel electrode secured over the contralateral frontal bone, and were simultaneously amplified ( $\times 2000$; NerveAmp, Center for Neural Recording, WA State University, Pullman, WA), analog filtered (bandpass cutoff $=-6 \mathrm{db}$ at $0.1-3000 \mathrm{~Hz}$, roll-off $=5 \mathrm{db} /$ octave) and digitized at $10 \mathrm{kHz}$. Evoked potentials were averaged over 120 stimulus presentations. Regions of auditory, somatosensory and visual cortex were estimated from interpolated (bicubic spline) maps of evoked potential amplitude across the recording array at select poststimulus latencies. The initial positive component $(\mathrm{P} 1)$ of the evoked potential complex occurred at the shortest poststimulus latency, and the center of its mapped amplitude peak was visually identified and used to locate regions of responsive cortex. Somatic stimulation was achieved by electrical stimulation. The hindpaw (HP), midtrunk (MT), forepaw (FP), were shaved and lightly coated with conductive jelly. A bipolar electrode (500 $\mu \mathrm{m}$ tips; $1 \mathrm{~mm}$ separation) attached to a constant current source delivered biphasic current pulses ( 1 $\mathrm{ms} ; 0.1-2 \mathrm{~mA})$ to the exposed skin. Auditory click stimuli $(0.1 \mathrm{~ms}$ monophasic pulses) were delivered using a high-frequency piezoelectric speaker placed $\sim 15 \mathrm{~cm}$ lateral to the contralateral ear. Visual stimulation (15 ms pulses) was delivered with an array of 5 high-intensity lightemitting diodes (white) positioned $\sim 2 \mathrm{~cm}$ from the contralateral eye.

$\mathrm{NeuN}$ immunohistochemical verification. After electrophysiological verification, brains were harvested and frozen $\left(-80^{\circ} \mathrm{C}\right) .30 \mu \mathrm{m}$ sections were taken throughout the lesion area and mounted on Superfrost-Plus slides (Fisher Scientific). Sections were then fixed for $1 \mathrm{~h}$ in $4 \%$ paraformaldehyde, washed $\left(6 \times 5 \mathrm{~min}\right.$ in PBS), treated with $0.3 \% \mathrm{H}_{2} \mathrm{O}_{0}$ solution and then washed and treated with an Avidin/Biotin Blocking Kit (Vector Laboratories). Tissue was washed and incubated with mouse-anti NeuN primary antibody (1:5000, MAB377 MSxNeuN; Millipore Bioscience Research Reagents) at $4^{\circ} \mathrm{C}$ for $48 \mathrm{~h}$ in a buffer consisting of $1 \%$ bovine serum albumin, $0.25 \%$ carrageen $\lambda$ and $5 \%$ Triton X-100 in PBS. After washes in PBS tissue was incubated for $2 \mathrm{~h}$ with biotinylated goat anti-mouse secondary antibody (1:200, Jackson ImmunoResearch), washed and incubated for $2 \mathrm{~h}$ with a standard ABC kit (Vectastain ABC Kit, Vector Laboratories). NeuN was visualized with $\mathrm{DAB}$ and nickel chromogens. Sections were coverslipped, the lesions were examined under a brightfield microscopy and neurons were identified as cells with black nuclei.

\section{Shock-sensitivity test}

Somatosensory reactivity to shock was observed in specialized chambers obtained commercially (Kinder Scientific). Each isolation chamber contained a rat holder (SM2001 Đ Kinder Scientific) held in place onto a platform load cell that detected cage displacement. Scrambled electric shock was administered through a metal grid (SMG-R Đ Kinder Scientific) connected to a programmable animal shocker (scrambled output; SMSCK Đ Kinder Scientific) inserted into each rat holder. A shocksensitivity gradient was determined by exposing rats to an increasing/ decreasing series of foot-shocks. Rats were placed into the holders and 50 ms shocks were administered in steps beginning at 0 (no shock) and increasing as follows: $0.04,0.10,0.14,0.2,0.24,0.3,0.34,0.4,0.44,0.5$, $0.54-0.60$ and then decreasing down to $0 \mathrm{~mA}$. Shocks were presented at a $60 \mathrm{~s}$ interval and the series was repeated twice with a 5-min interseries interval. Sensitivity to shock was inferred by average displacement of the load cell (shock elicited startle) averaged across a total of 4 trials at each shock intensity. Displacement was recorded by a controlling computer for $200 \mathrm{~ms}$ beginning with the initial presentation of each electric shock and converted to force $(N)$ using known standards.

\section{Experimental designs}

Social exploration. To determine the efficacy of a $5 \mathrm{~s}$ chamber blackout as a safety signal in reducing persistent stress-induced anxiety rats were randomly assigned to the following treatments ( $n$ s $=8$ /group) as described above: Safe, Random, Variable, Fixed, HC-Random, or HC. In the primary experimental condition, Safe, a $5 \mathrm{~s}$ chamber blackout began with the termination of each shock. All rats were given a 3 min social exploration test $24 \mathrm{~h}$ after stress.

Dorsal raphe nucleus. Activation of the caudal DRN is a necessary component of the effects of inescapable tail shock on numerous behavioral endpoints including social exploration (Christianson et al., 2008) (for review see Maier and Watkins, 2005). Controllable stress is associated with inhibition of caudal DRN activity (Maswood et al., 1998; Grahn et al., 1999; Amat et al., 2005). Importantly, all regions of the serotonergic raphé are activated by tail-shock stress (Takase et al., 2004), but only caudal DRN activity is critical to the behavioral consequences (Hammack et al., 2002). Therefore it was likely that, as with behavioral control, safety signals might mitigate the effects of stress by recruiting inhibitory input to the caudal DRN resulting in lesser activation. Rats were cannulated for in vivo microdialysis in the DRN. 5-HT efflux within the DRN is a measure of DRN 5-HT activity because activated DRN neurons release 5-HT within the DRN from axon collaterals, as well as in projection regions (Matos et al., 1996). Dialysis samples were collected every $20 \mathrm{~min}$ before (B1-B4), during (S1-S4) and after (P1-P4) Safe or Random shock ( $n \mathrm{~s}=7 /$ group, 12 samples/rat). Each subject served as its own control and unstressed rats were not included because previous work has repeatedly indicated that neither $\mathrm{HC}$ nor restraint in tubes produces an elevation in DRN 5-HT (Maswood et al., 1998). In any case, the critical question was simply whether the presence of a safety signal would reduce the DRN 5-HT increase produced by tail shock. Because Random did not differ from Variable in its behavioral effects above, this was the correct comparison group. A second set of rats were exposed to either Safe, Random or HC treatment $(n \mathrm{~s}=8)$ and $2 \mathrm{~h}$ after the onset of stress were killed, perfused and processed for colocalization of 5-HT and Fos, the protein product of the immediate early gene $c$-fos and a marker for neuronal activity. Here a $\mathrm{HC}$ group was necessary because animals could not be compared with their own baseline before stress.

Ventromedial prefrontal cortex. Activity within the vmPFC is critical to numerous effects of behavioral control on later behaviors (Amat et al., 2005, 2006; Baratta et al., 2007; Rozeske et al., 2008) including social exploration (Christianson et al., 2008b). Although safety signals did not appear to inhibit the DRN as does behavioral control, it remained possible that some cognitive aspect of safety would recruit the vmPFC. Therefore, rats were cannulated for microinjection of the $\mathrm{GABA}_{\mathrm{A}}$ agonist muscimiol into the vmPFC using identical methods previously described (Christianson et al., 2008b). After 7-10 d of recovery all rats were given a social exploration pretest. On the next day rats were randomly assigned to either muscimol or saline injection and either Safe or Random tail shock in a $2 \times 2$ factorial design ( $n=8$ /group). Microinjections were made $60 \mathrm{~min}$ before the onset of stress. Social exploration was tested $24 \mathrm{~h}$ later.

Sensory insular lesions and safety signals. Because of its anatomical configuration and electrophysiological properties, $\mathrm{Si}$ is situated to contribute to processes related to associative learning (Rodgers et al., 2008), and, by extension, the processing of safety. Bilateral excitotoxic lesions or sham surgeries were made to the Si using anatomical landmarks on the surface of neocortex that have been previously defined using highresolution field potential mapping (see (Rodgers et al., 2008) for extensive descriptions). After 2 weeks of recovery, each rat received a social exploration baseline test; lesion treatment had no effect on social exploration. Rats were then exposed to either Safe or Random shock and social exploration was tested again $24 \mathrm{~h}$ later. Thus, the design of this experiment is a $2 \times 2$ factorial ( $n=8$ /group). To control for possible somatosensory processing interference by the $\mathrm{Si}$ lesion, all rats were assessed for sensitivity to foot-shock. The specificity of the excitotoxic lesions was then assessed in two ways. First, each rat was anesthetized and after bilateral craniotomy, hemispheric mapping of the epipial somotosensory evoked potential (SEP), auditory evoked potential (AEP), and visual evoked potentials (VEP) were performed. Rats that showed any SEP, AEP or VEP in the Si region were considered incomplete lesions. In addition, rats that exhibited a change in evoked potentials (amplitude, wave morphology or areal distribution) in primary or secondary somatosensory, 
auditory or visual cortex because of lesion spread were excluded. This approach insured that the functional responsiveness of the Si target region was selectively and fully lesioned, and confirmed that the lesioned animals had no damage to primary visual cortex necessary for light detection of the safety signal. Second, representative coronal sections were stained for NeuN, a neuron-selective antibody. Rats were included in the behavioral analysis if there was no response to SEP, AEP and VEP and if histological verification revealed neuronal loss restricted to the target region.

Sensory insular lesions and behavioral control. Behavioral control activates the vmPFC, which in turn provides inhibitory input to the DRN (Amat et al., 2005). Furthermore, vmPFC activation and DRN 5-HT inhibition are both necessary to the protective effects of control on social exploration (Christianson et al., 2008). Because safety signals exert their effects without modulating the DRN and without activating the vmPFC during stress, these structures are unlikely to mediate the processing of safety signals. However, if generation of safety signals is a component of behavioral control, then Si lesions should interfere with the protective effects conferred by control. Thus, bilateral Si lesions were made as above and after recovery rats were exposed to 100 trials of ESs, yoked ISs of equal duration, or left in the home cage (HC; $n=8$ /group). Social exploration tests were conducted $24 \mathrm{~h}$ before and after stress and lesions were verified as above.

\section{Statistical methods}

Behavioral data were analyzed with ANOVA with stress and lesion treatments as between-subjects variables and shock intensity as a withinsubjects variable. The assumption of homogeneity of variance (or sphericity for within-subjects tests) was tested before each analysis with the Bartlett test of equal variance or the Mauchly test of sphericity. In all cases the data were deemed appropriate for parametric analysis. Post hoc pairwise comparisons were made using Tukey's least squared difference method to control the experiment-wise type-I error rate at $\alpha=0.05$.

\section{Results}

\section{Social exploration}

The mean time spent investigating the juvenile is shown in Figure 1B. Tail shock, whether of Fixed or Variable duration, reduced social investigation $24 \mathrm{~h}$ later. The presence of $5 \mathrm{~s}$ blackouts that were randomly related to the shocks (Random) did not reduce this effect. However, $5 \mathrm{~s}$ blackouts that consistently followed each shock (Safe) completely blocked this effect of tail shock. One-way ANOVA identified a significant effect of group $F_{(5,42)}=10.18, p<$ 0.001 . Subsequent pairwise comparisons identified significant differences between Safe and all other groups receiving shock, which all differed from each HC group but not from each other, $p s<0.05$.

\section{Dorsal raphe nucleus serotonin}

Tail shock produced a large increase in extracellular 5-HT (Fig. $2 A$ ), but surprisingly, this was not reduced by the presence of the safety signal, even though this condition had blocked the behavioral effects of tail shock. Indeed, extracellular 5-HT levels were actually increased more by shock in group Safe than Random, although the difference was not statistically significant. Extracellular 5-HT was computed as the percentage of the mean of the first 4 baseline samples. Repeated-measures ANOVA revealed a significant effect of sample, $F_{(11,99)}=20.06, p<0.001$ but no effect of group or group by time interaction, $p>0.30$. Pos hoc tests identified that samples S1, S2, S3, S4, P1, P2, and P3 were significantly greater than baseline samples in both Safe and Random groups, $p<0.05$. The Fos data were consistent with this pattern. Tail shock induced Fos in 5-HT labeled neurons in the caudal DRN in both Safe and Random groups (Fig. 2 B). One-way ANOVA revealed a significant main effect of stress on the percentage of 5-HT-positive cells containing Fos, $F_{(2,20)}=12.46, p<0.001$ with significantly more staining in both Safe and Random groups compared with HC, $p \leq 0.01$. Safe and Random did not differ from each other. Because activation of the DRN is equal under safety signal and
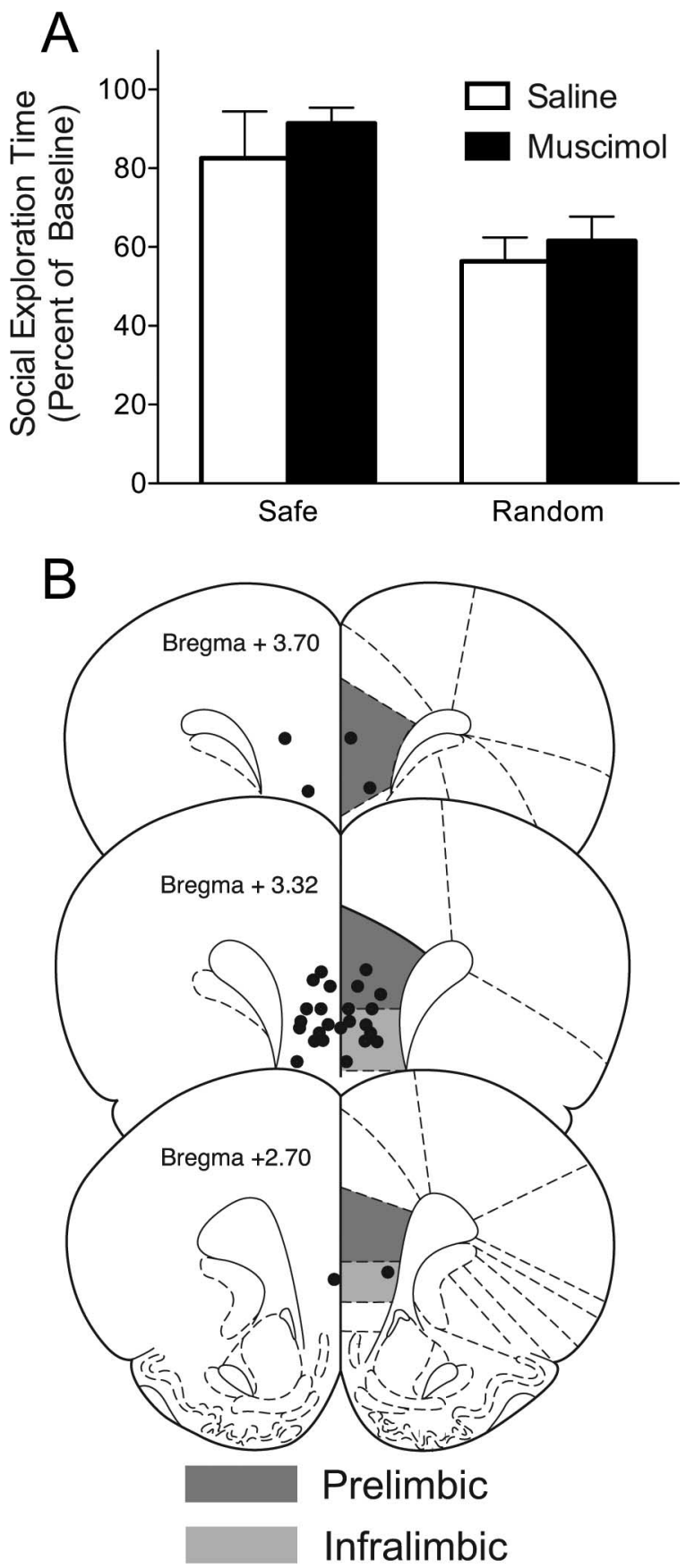

Figure 3. Effect of ventromedial prefrontal cortex inactivation during tail shock with safe or random signals on social exploration $24 \mathrm{~h}$ later. $\boldsymbol{A}$, Mean (+SEM) time spent exploring the juvenile conspecific expressed as percentage of baseline. $\boldsymbol{B}$, Illustration of microinjector cannula tips throughout the prelimbic and infralimbic subregions of the vmPFC. Anatomical maps adapted from the atlas of Paxinos and Watson (1998).

random signal shock, but the behavioral consequences are different, the results indicate that safety signals exert their effect over stress in a way that is distinct from that of behavioral control.

\section{Ventromedial prefrontal cortex inhibition}

All rats included in the behavioral analysis were found to have microinjector tips located within either the infralimbic or prelimbic region of the vmPFC (Fig. 3B). Half of the subjects in the critical experimental group Safe-Muscimol had cannula placements within the infralimbic region whereas the other half were 

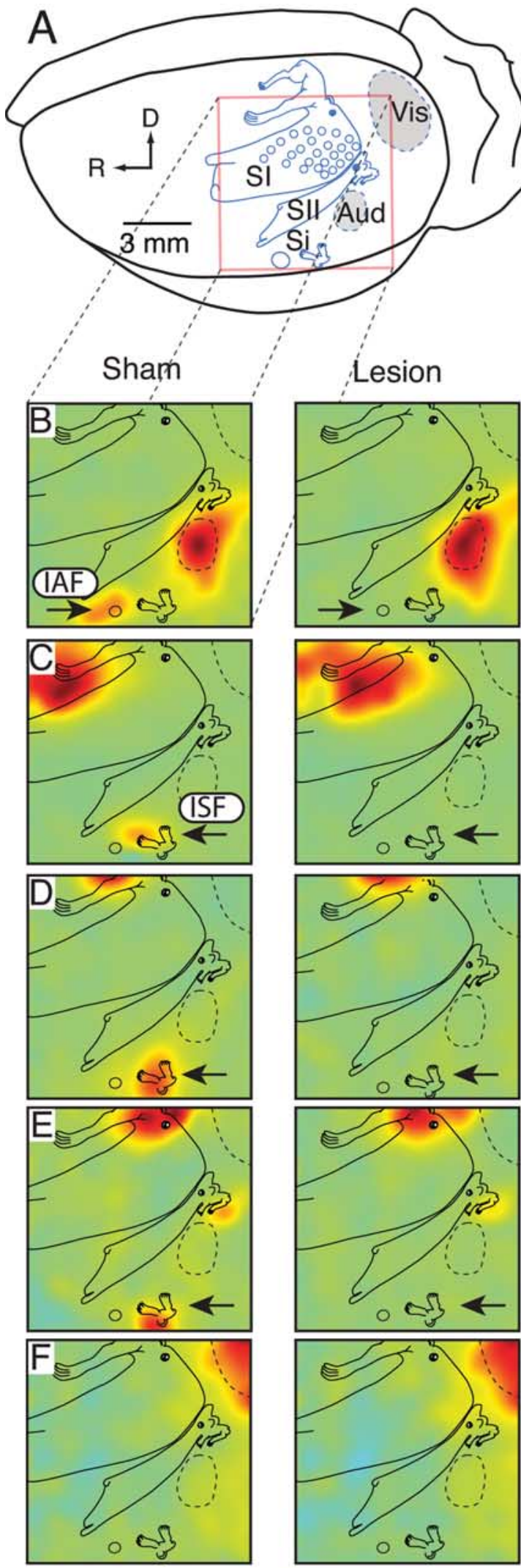

$-100 \%$

Figure 4. Electrophysiological verification of Si lesions. $A, A$ ratunculus derived from more extensive mapping studies has been superimposed on the left hemisphere as a functional/ anatomical reference, depicting the somatotopic organization and location of primary and secondary somatosensory cortex (SI and SII, respectively) including the easily recognized whisker representations (small circles). The insular auditory and somatosensory fields (IAF and ISF)

in the prelimbic region. Importantly, social exploration time was equal in these subgroups. In this experiment each rat served as its own control and social exploration is expressed as the percentage of baseline. The safety signal reduced the impact of tail shock on later social investigation but inhibition of the vmPFC with muscimol had no effect (Fig. 3A). A two-way ANOVA identified a main effect of Stress, $F_{(1,23)}=13.03, p=0.002$, but the effect of Drug and the Stress by Drug interaction did not reach significance, $p>0.37$.

\section{Sensory insular lesions and safety signals}

All rats included in the behavioral analysis were found to have complete but selective lesions to the Si. The hemispheric maps of evoked potentials from a representative lesion and sham rat are depicted in Figure 4 and a representative photomicrograph of NeuN immunoreactivity is depicted in Figure 5. No difference was found in basal social exploration between lesion and sham treatments, means $(\mathrm{SEM})$ : Sham $=89.91$ (3.39), Lesion $=88.35$ (3.49), $t_{(30)}=0.320, p=0.751$. Again, each rat served as its own control and social exploration is expressed as the percentage of baseline. As before, the safety signal reduced the impact of tail shock on later social investigation. Importantly, this protective effect was completely blocked by Si lesion (Fig. 6A). A two-way ANOVA identified a main effect of Lesion, $F_{(1,26)}=6.63, p=$ 0.032 and a significant Stress by Lesion interaction, $F_{(1,26)}=5.14$, $p=0.016$. Pairwise comparisons found that all groups were significantly lower than the Safe/Sham group, $p<0.05$, and did not differ among themselves. In the shock sensitivity test Lesion and Sham groups displayed equal sensitivity to grid shocks of increasing intensity (Fig. 6B). A two-way ANOVA identified a significant main-effect of shock intensity, $F_{(11,330)}=117.829, p<0.001$ but no effect of lesion group or the interaction, $p>0.8$.

\section{Sensory insular lesions and behavioral control}

All subjects were found to have complete and selective lesions of the Si. Lesion treatment had no effect on baseline social exploration times, means (SEM): Sham $=88.85(1.72)$, Lesion $=87.67$ $(2.46), t_{(46)}=0.392, p=0.697$. This critical experiment would be impossible if Si lesions were to interfere with acquisition of the wheel-turn escape response. Wheel-turn escape latencies were averaged into 10 trial blocks for each rat. Although a two-way ANOVA revealed a significant effect of lesion, $F_{(1,70)}=7.392, p=$ 0.008 it is clear that rats in both Lesion and Sham groups learned the escape response (Fig. 7A). Post hoc contrasts between lesion groups on each trial block identified a significant difference only for block $2, p=0.048$. The potential for this group difference to impact later behavior is moot because $\mathrm{Si}$ lesions did interact with the effects of control on social exploration (Fig. 7B). Regardless of lesion treatment, IS reduced social exploration whereas ES com-

\footnotetext{
$\leftarrow$

are near the rhinal sulcus and referred to together as sensory insula (Si). Auditory (Aud) and visual (Vis) cortices are also shown. The location and extent of the $6 \times 6 \mathrm{~mm}$ recording array (red box) is shown in relation to these cortical areas. R and D reflect rostral and dorsal, respectively. $\boldsymbol{B}$, In sham animals (left column) an interpolated amplitude map of the earliest positive peak of the auditory evoked potential (AEP) showed maximum activity in auditory cortex with a separate island of activity in IAF (arrow). After lesion (right column), activity in auditory cortex remained the same but that of IAF was completely eliminated (arrow). C-E, Similar maps of somatosensory evoked potentials (SEP) from forepaw, hindpaw, and midtrunk stimulation, respectively. Activation of ISF in the lesion animals was eliminated after lesions to Si (arrows). Note that clear activation of the midtrunk representation in SII remained intact, demonstrating the specificity of the Si lesions. $\boldsymbol{F}$, Visual evoked potentials (VEP) remained unchanged by the lesion. All maps were normalized to the largest amplitude response for a given condition and are thus represented as a percentage of maximum within each map.
} 

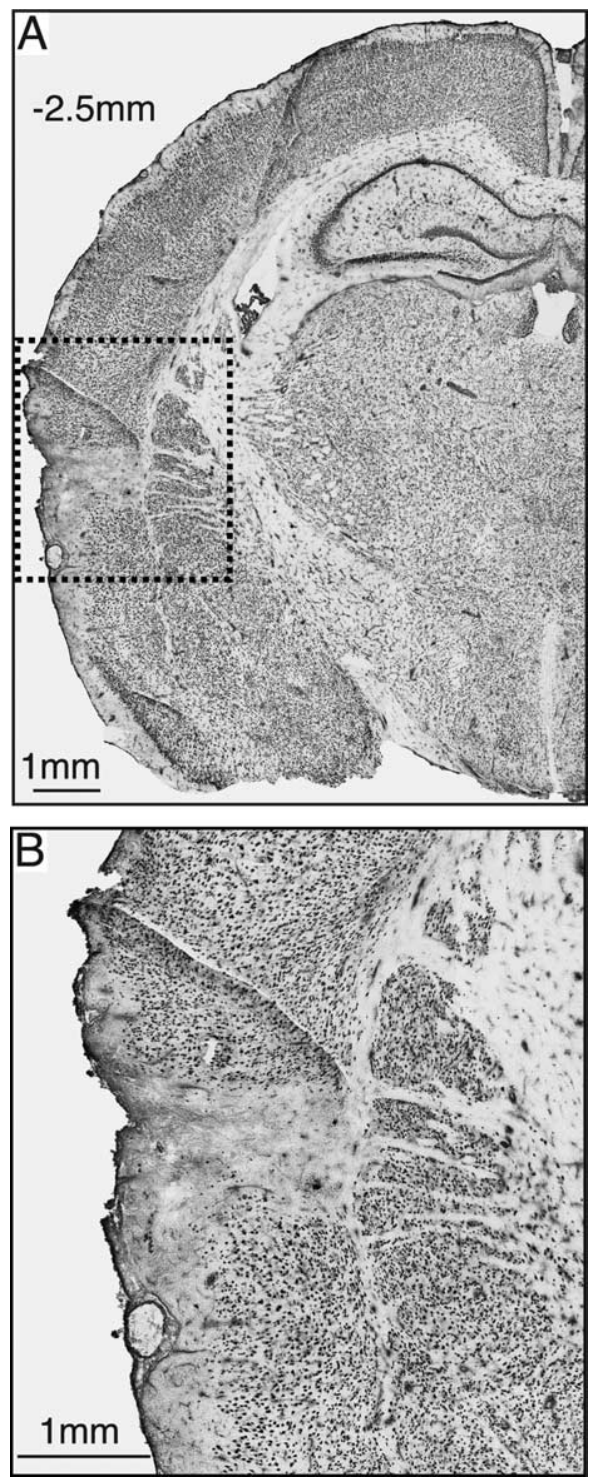

Figure 5. Immunohistochemical lesion verification. $\boldsymbol{A}$, NeuN immunoreactivity in a typical coronal section at bregma $-2.5 \mathrm{~mm}$ (center of $\mathrm{Si}$ ) showed selective neuronal death confined to an $\sim 1 \mathrm{~mm}$ area (dashed box). $\boldsymbol{B}$, An enlargement of the lesion within the dashed box reveals that neuronal death was confined to Si and did not cross into the striatum.

pletely prevented this effect. A two-way ANOVA identified a main-effect of Stress, $F_{(2,40)}=24.30, p<0.001$ but no effect of Lesion or a Stress by Lesion interaction, $p>0.7$. Post hoc comparisons revealed that IS significantly reduced social exploration compared with both ES and HC, which did not differ, $p<0.001$.

\section{Discussion}

The provision of a signal immediately after the termination of each shock eliminated the reduction in social exploration caused by inescapable tail shock observed $24 \mathrm{~h}$ later, just as we have observed with behavioral control (Christianson et al., 2008). This finding is consistent with that of Jackson and Minor (1988) and Minor et al. (1990), who reported that similar safety signals eliminated the shuttlebox escape deficits that normally follow tail shock, an effect also blocked by behavioral control. Here we extend the effect of a safety signal to a behavior that does not involve shock at all, juvenile social investigation, a behavior that may reflect anxiety (File and Seth, 2003). It is not obvious why a $5 \mathrm{~s}$ stimulus occurring at the end of each shock reduces the impact of the stressor on later behavior, as the stimulus does not provide the organism with any temporal information about the occurrence of the next shock beyond that provided by the termination of the preceding shock itself. That is, the safety signal does not aid in the prediction of when shocks will occur in any obvious way. However, a stimulus that regularly follows each stressor does acquire the ability to inhibit fear while it is present. Perhaps the presence of an explicit conditioned inhibitor of fear reduces the total time spent in fear, a suggestion made by Jackson and Minor (1988). It might be noted that the effects of a safety signal on later behaviors after tail shock do not depend on the use of a blackout as the cue (Maier and Keith, 1987).

That the provision of a safety signal mimicked the effects of behavioral control suggested that perhaps pavlovian signaling features of the controlling escape response underlie the impact of control. However, the experiments reported here indicate that safety signals and control use different mechanisms to blunt the behavioral impact of the stressor. The presence of the safety signal did not reduce the DRN 5-HT activation produced by the stressor, as does control. There was not even a tendency for the safety signal to have such an effect, either with Fos in 5-HT-labeled cells or extracellular 5-HT within the DRN as the measure. Furthermore, inhibition of the vmPFC with muscimol, a procedure that abolished the effect of behavioral control on both behavior and DRN regulation (Amat et al., 2005), had no effect on safety.

These results led us to explore the involvement of brain regions that are situated to detect the coincidence of stimuli, in this case a chamber blackout and a tail shock, and to compute under which environmental circumstances to inhibit fear. Indeed, the Si receives sensory input required for this calculation (Benison et al., 2007; Rodgers et al., 2008) and interacts with the amygdala (McDonald et al., 1999), a region involved in fear conditioning (Kim and Jung, 2006). Here, Si lesions completely blocked the impact of the safety signal. Contrary to the hypothesis that learned safety is a component of behavioral control, Si lesions had no effect on the protective effect of control, thereby providing a double-dissociation. Thus, it would appear that the operation of behavioral control cannot be reduced to a special case of the operation of safety cues and that safety cues mimic only the behavioral outcome of control and not the neural substrate. Clearly, there are multiple mechanisms by which psychological factors can modulate the impact of stressors on later behavior.

Although there is a great deal known concerning the neural mechanisms by which behavioral control modulates the impact of stressors, the mechanisms by which safety signals acquire the capacity to inhibit fear or to modulate later behavior are not well understood. Indeed, the published results are largely negative. Thus, it has been reported that nucleus accumbens (Josselyn et al., 2005), central nucleus of the amygdala (Falls and Davis, 1995), prefrontal cortex (Gewirtz et al., 1997), or perirhinal (Falls et al., 1997) lesions do not interfere with the acquisition and/or expression of conditioned fear inhibition by signals that are unpaired with shock. Although posttraining lesions of the hippocampus do reduce the ability of conditioned inhibitors to reduce fear, further training overcomes this effect and pretraining lesions do not reduce the acquisition of fear inhibitory properties by safety signals (Heldt and Falls, 2006). Here we did not measure the ability of the safety signal to inhibit fear, but rather whether the safety signal would blunt the impact of the stressor on later behavior. Nevertheless, a pretraining Si lesion completely blocked the effects of the safety signal. This is the first report of a pretraining lesion of a limbic or cortical structure to block a safety 

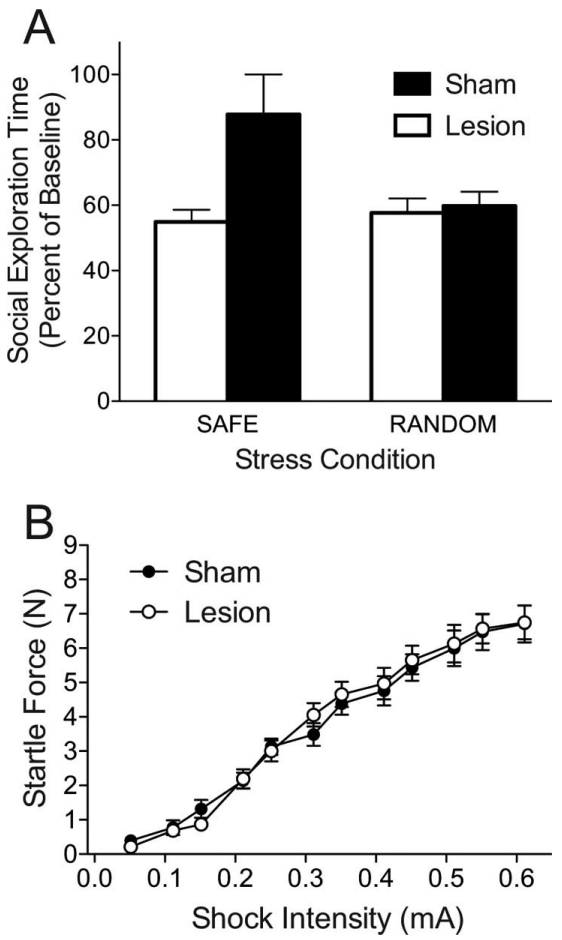

Figure 6. Effect of bilateral excitotoxic Si lesions on social exploration $24 \mathrm{~h}$ after tail shock with safe or random signals. $\boldsymbol{A}$, Mean (+SEM) time spent exploring the juvenile conspecific expressed as percentage of baseline. $\boldsymbol{B}$, Mean ( \pm SEM) startle force in response to in response to foot shocks of increasing intensity.

signal effect. However, the present data do not indicate whether the lesion: (1) blocked the formation of the association between the signal and the absence of shock, i.e., acquisition of conditioned inhibition of fear, or (2) did not hinder formation of the association, but instead blunted the impact of the established signal on the neurochemical sequelae of tail shock that lead to the persistent behavioral changes. Additional studies are required to determine whether $\mathrm{Si}$ is critically involved in more traditional conditioned inhibition learning. However, the anatomical organization of Si may inform its role in the processing of safety signals.

Rogan et al. (2005) observed physiological responses in the striatum that were correlated with the presentation of safety signals but were distinct from fear cues and, in the same paradigm, safety cues appeared to blunt the typical response patterns of neurons in the lateral amygdala evoked by danger signals. The current results suggest that the Si may participate with the striatum and/or the amygdala to process safety signals. The Si in many species receives multisensory input that includes somatosensory, auditory and visual information (Loe and Benevento, 1969; Benedek et al., 1986; Cechetto and Saper, 1987; Benedek and Hicks, 1988; Hanamori et al., 1998; Hicks et al., 1988a,b; Zhang and Oppenheimer, 2000; Bamiou et al., 2003; Rodgers et al., 2008) and so it is well suited to develop the association or contingency between the signal and the absence of the stressor. Indeed when presented with stimuli from different modalities, the Si exhibits superlinear evoked potentials which suggest multisensory processing (Rodgers et al., 2008). Interestingly, the vmPFC and its associated striatal projections have been implicated in the acquisition of response-outcome contingencies (for review, see Yin and Knowlton, 2006; Balleine et al., 2007). The Si projects to the striatum [retrograde data, Si referred to as PV (Alloway et al., 2006), and anterograde data (A. Benison, unpublished data)] and
A
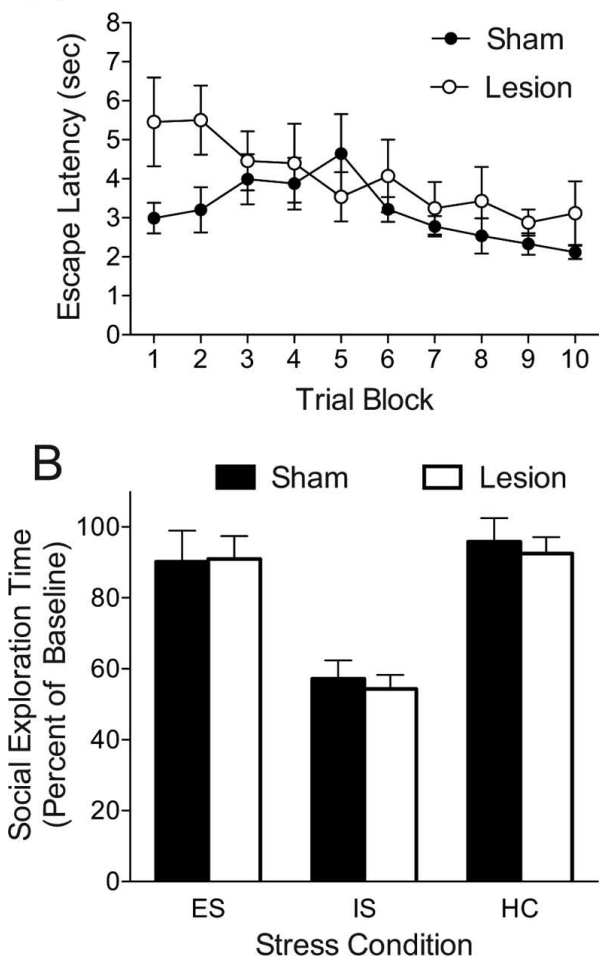

Figure 7. Effect of bilateral excitotoxic Si lesion on wheel-turn escape performance and social exploration $24 \mathrm{~h}$ after escapable, yoked inescapable, or no shock. $\boldsymbol{A}$, Mean ( \pm SEM) latency to perform wheel-turn escape requirement during escapable shock. Trial blocks represent 10 trials. $\boldsymbol{B}$, Mean (+SEM) time spent exploring the juvenile conspecific expressed as percentage of baseline.

so it is possible that there is an Si-striatal circuit that processes stimulus-stimulus contingencies that is analogous to the vmPFCdorsal striatal circuit that processes response-outcome contingencies. Although Rogan et al. (2005) suggest that striatum is involved in positive affective aspects of safety, the actual contribution of striatum to these effects remains open. Si-amygdala projections have also been reported (Shi and Cassell, 1998a,b; McDonald et al., 1999) and it is possible that this pathway is important to the inhibition of fear associated with safety. Thus, in concert with striatum and amygdala the Si may play a critical, and previously unrecognized, role in stimulus-stimulus associative learning and fear inhibition.

\section{References}

Alloway KD, Lou L, Nwabueze-Ogbo F, Chakrabarti S (2006) Topography of cortical projections to the dorsolateral neostriatum in rats: multiple overlapping sensorimotor pathways. J Comp Neurol 499:33-48.

Amat J, Baratta MV, Paul E, Bland ST, Watkins LR, Maier SF (2005) Medial prefrontal cortex determines how stressor controllability affects behavior and dorsal raphe nucleus. Nat Neurosci 8:365-371.

Amat J, Paul E, Zarza C, Watkins LR, Maier SF (2006) Previous experience with behavioral control over stress blocks the behavioral and dorsal raphe nucleus activating effects of later uncontrollable stress: role of the ventral medial prefrontal cortex. J Neurosci 26:13264-13272.

Balleine BW, Delgado MR, Hikosaka O (2007) The role of the dorsal striatum in reward and decision-making. J Neurosci 27:8161-8165.

Bamiou DE, Musiek FE, Luxon LM (2003) The insula (Island of Reil) and its role in auditory processing. Literature review. Brain Res Brain Res Rev 42:143-154.

Baratta MV, Christianson JP, Gomez DM, Zarza CM, Amat J, Masini CV, Watkins LR, Maier SF (2007) Controllable versus uncontrollable stressors bi-directionally modulate conditioned but not innate fear. Neuroscience 146:1495-1503. 
Benedek G, Hicks TP (1988) The visual insular cortex of the cat: organization, properties and modality specificity. Prog Brain Res 75:271-278.

Benedek G, Jang EK, Hicks TP (1986) Physiological properties of visually responsive neurones in the insular cortex of the cat. Neurosci Lett 64:269-274.

Benison AM, Rector DM, Barth DS (2007) Hemispheric mapping of secondary somatosensory cortex in the rat. J Neurophysiol 97:200-207.

Cechetto DF, Saper CB (1987) Evidence for a viscerotopic sensory representation in the cortex and thalamus in the rat. J Comp Neurol 262:27-45.

Christianson JP, Paul ED, Irani M, Thompson BM, Kubala KH, Yirmiya R, Watkins LR, Maier SF (2008a) The role of prior stressor controllability and the dorsal raphe nucleus in sucrose preference and social exploration. Behav Brain Res 193:87-93.

Christianson JP, Thompson BM, Watkins LR, Maier SF (2008b) Medial prefrontal cortical activation modulates the impact of controllable and uncontrollable stressor exposure on a social exploration test of anxiety. Stress, in press.

Falls WA, Davis M (1995) Lesions of the central nucleus of the amygdala block conditioned excitation, but not conditioned inhibition of fear as measured with the fear-potentiated startle effect. Behav Neurosci 109:379-387.

Falls WA, Bakken KT, Heldt SA (1997) Lesions of the perirhinal cortex interfere with conditioned excitation but not with conditioned inhibition of fear. Behav Neurosci 111:476-486.

File SE, Seth P (2003) A review of 25 years of the social interaction test. Eur J Pharmacol 463:35-53.

Flynn FG (1999) Anatomy of the insula functional and clinical correlates. Aphasiology 13:55-78.

Gewirtz JC, Falls WA, Davis M (1997) Normal conditioned inhibition and extinction of freezing and fear-potentiated startle following electrolytic lesions of medical prefrontal cortex in rats. Behav Neurosci 111:712-726.

Grahn RE, Will MJ, Hammack SE, Maswood S, McQueen MB, Watkins LR, Maier SF (1999) Activation of serotonin-immunoreactive cells in the dorsal raphe nucleus in rats exposed to an uncontrollable stressor. Brain Res 826:35-43.

Hammack SE, Richey KJ, Schmid MJ, LoPresti ML, Watkins LR, Maier SF (2002) The role of corticotropin-releasing hormone in the dorsal raphe nucleus in mediating the behavioral consequences of uncontrollable stress. J Neurosci 22:1020-1026.

Hanamori T, Kunitake T, Kato K, Kannan H (1998) Responses of neurons in the insular cortex to gustatory, visceral, and nociceptive stimuli in rats. J Neurophysiol 79:2535-2545.

Heldt SA, Falls WA (2006) Posttraining lesions of the auditory thalamus, but not cortex, disrupt the inhibition of fear conditioned to an auditory stimulus. Eur J Neurosci 23:765-779.

Hicks TP, Benedek G, Thurlow GA (1988a) Organization and properties of neurons in a visual area within the insular cortex of the cat. J Neurophysiol 60:397-421.

Hicks TP, Benedek G, Thurlow GA (1988b) Modality specificity of neuronal responses within the cat's insula. J Neurophysiol 60:422-437.

Jackson RL, Minor TR (1988) Effects of signaling inescapable shock on subsequent escape learning: implications for theories of coping and "learned helplessness". J Exp Psychol Anim Behav Process 14:390-400.

Josselyn SA, Falls WA, Gewirtz JC, Pistell P, Davis M (2005) The nucleus accumbens is not critically involved in mediating the effects of a safety signal on behavior. Neuropsychopharmacology 30:17-26.

Kim JJ, Jung MW (2006) Neural circuits and mechanisms involved in Pavlovian fear conditioning: a critical review. Neurosci Biobehav Rev 30:188-202.

Loe PR, Benevento LA (1969) Auditory-visual interaction in single units in the orbito-insular cortex of the cat. Electroencephalogr Clin Neurophysiol 26:395-398.

Maier SF, Keith JR (1987) Shock signals and the development of stressinduced analgesia. J Exp Psychol Anim Behav Process 13:226-238.

Maier SF, Watkins LR (1998) Stressor Controllability, Anxiety, and Serotonin. Cognitive Therapy and Res 22:595-613.

Maier SF, Watkins LR (2005) Stressor controllability and learned helplessness: the roles of the dorsal raphe nucleus, serotonin, and corticotropinreleasing factor. Neurosci Biobehav Rev 29:829-841.

Maier SF, Rapaport P, Wheatley KL (1976) Conditioned inhibition and the UCS-CS interval. Anim Learn Behav 4:217-220.
Maier SF, Amat J, Baratta MV, Paul E, Watkins LR (2006) Behavioral control, the medial prefrontal cortex, and resilience. Dialogues Clin Neurosci 8:397-406.

Maswood S, Barter JE, Watkins LR, Maier SF (1998) Exposure to inescapable but not escapable shock increases extracellular levels of 5-HT in the dorsal raphe nucleus of the rat. Brain Res 783:115-120.

Matos FF, Urban C, Yocca FD (1996) Serotonin (5-HT) release in the dorsal raphe and ventral hippocampus: raphe control of somatodendritic and terminal 5-HT release. J Neural Transm 103:173-190.

McDonald AJ, Shammah-Lagnado SJ, Shi C, Davis M (1999) Cortical afferents to the extended amygdala. Ann N Y Acad Sci 877:309-338.

Mineka S, Hendersen RW (1985) Controllability and predictability in acquired motivation. Annu Rev Psychol 36:495-529.

Mineka S, Cook M, Miller S (1984) Fear conditioned with escapable and inescapable shock: effects of a feedback stimulus. J Exp Psychol Anim Behav Process 10:307-323.

Minor TR, Hunter AM (2002) Stressor controllability and learned helplessness research in the United States: sensitization and fatigue processes. Integr Physiol Behav Sci 37:44-58.

Minor TR, Trauner MA, Lee CY, Dess NK (1990) Modeling signal features of escape response: effects of cessation conditioning in "learned helplessness" paradigm. J Exp Psychol Anim Behav Process 16:123-136.

Mufson EJ, Mesulam MM (1982) Insula of the old world monkey. II. Afferent cortical input and comments on the claustrum. J Comp Neurol 212:23-37.

Paxinos G, Watson G (1998) The rat brain in stereotaxic coordinates, Ed 4. New York: Academic.

Remple MS, Henry EC, Catania KC (2003) Organization of somatosensory cortex in the laboratory rat (Rattus norvegicus): evidence for two lateral areas joined at the representation of the teeth. J Comp Neurol 467:105-118.

Robinson CJ, Burton H (1980a) Somatic submodality distribution within the second somatosensory (SII), 7b, retroinsular, postauditory, and granular insular cortical areas of M. fascicularis. J Comp Neurol 192:93-108.

Robinson CJ, Burton H (1980b) Organization of somatosensory receptive fields in cortical areas $7 \mathrm{~b}$, retroinsula, postauditory and granular insula of M. fascicularis. J Comp Neurol 192:69-92.

Robinson CJ, Burton H (1980c) Somatotopographic organization in the second somatosensory area of M. fascicularis. J Comp Neurol 192:43-67.

Rodgers KM, Benison AM, Klein A, Barth DS (2008) Auditory, somatosensory, and multisensory insular cortex in the rat. Cereb Cortex 18:2941-2951.

Rogan MT, Leon KS, Perez DL, Kandel ER (2005) Distinct neural signatures for safety and danger in the amygdala and striatum of the mouse. Neuron 46:309-320.

Rozeske RR, Der-Avakian A, Bland ST, Beckley JT, Watkins LR, Maier SF (2008) The medial prefrontal cortex regulates the differential expression of morphine-conditioned place preference following a single exposure to controllable or uncontrollable stress. Neuropsychopharmacology, in press.

Shi CJ, Cassell MD (1998a) Cascade projections from somatosensory cortex to the rat basolateral amygdala via the parietal insular cortex. J Comp Neurol 399:469-491.

Shi CJ, Cassell MD (1998b) Cortical, thalamic, and amygdaloid connections of the anterior and posterior insular cortices. J Comp Neurol 399:440-468.

Sudakov K, MacLean PD, Reeves A, Marino R (1971) Unit study of exteroceptive inputs to claustrocortex in awake, sitting, squirrel monkey. Brain Res 28:19-34.

Takase LF, Nogueira MI, Baratta M, Bland ST, Watkins LR, Maier SF, Fornal CA, Jacobs BL (2004) Inescapable shock activates serotonergic neurons in all raphe nuclei of rat. Behav Brain Res 153:233-239.

Weiss JM (1971) Effects of coping behavior with and without a feedback signal on stress pathology in rats. J Comp Physiol Psychol 77:22-30.

Yin HH, Knowlton BJ (2006) The role of the basal ganglia in habit formation. Nat Rev Neurosci 7:464-476.

Zhang ZH, Oppenheimer SM (2000) Baroreceptive and somatosensory convergent thalamic neurons project to the posterior insular cortex in the rat. Brain Res 861:241-256. 